\title{
A Promising Target in Rheumatoid Arthritis Treatment: Granulocyte-Macrophage Colony-Stimulating Factor
}

\author{
A. Berkant Avci, MD ${ }^{1, *}$ \\ Eugen Feist, $M D^{2}$ \\ Gerd-R. Burmester, $M D^{2}$ \\ Address \\ ${ }^{*}, 1$ Division of Rheumatology, Department of Internal Medicine, Akdeniz University \\ Medical School, Antalya, Turkey \\ Email: avcialiberkant@yahoo.com \\ ${ }^{2}$ Department of Rheumatology and Clinical Immunology, Charite-University Med- \\ icine Berlin, Berlin, Germany
}

Published online: 20 0ctober 2015

(C) Springer International Publishing AG 2015

This article is part of the Topical Collection on Rheumatoid Arthritis

Keywords Rheumatoid arthritis · Treatment · GM-CSF · Mavrilimumab · MOR103 · Namilumab

\section{Opinion statement}

Granulocyte-macrophage colony-stimulating factor (GM-CSF) is more familiar to clinicians as a hemopoietic growth factor. However, it also modulates functions of myeloid cells such as macrophages. Experimental work has demonstrated its significant contribution to the pathogenesis of rheumatoid arthritis (RA). It was a long-time concern that targeted therapies against this cytokine could cause severe side effects such as neutropenia or pulmonary alveolar proteinosis. Nevertheless, different compounds successfully entered clinical development for RA targeting the cytokine itself or its receptor. Currently, a monoclonal antibody against its receptor has completed phase II trials with a profound and rapid onset of response, normalization of acute phase reactants, and an overall good safety profile. The development of compounds targeting the GM-CSF pathway represents a promising approach in RA. However, the obtained ACR20 and ACR50 responses were rather similar with marketed biologic agents when added to MTX. So, it is appropriate to ask the question, whether we do really need another group of agents with a similar performance? In this context, the tumor necrosis factor (TNF)-independent mode-of action of GM-CSF blockade supports the assumption that it could be a useful alternative especially in anti-TNF-resistant patients. 
Furthermore, a higher efficacy with concurrent blockade of IL-17 and GM-CSF, as reported from preclinical studies, also suggests that such strategies are of interest for future approaches. Therefore, comprehensive assessment of GM-CSF blockade in antiTNF-resistant patients and combination strategies will be of major importance. Taken together, rapid onset of action, sustained effectiveness, and shown favorable safety data from phase 2 trials warrant further evaluations of anti-GM-CSF agents in different subgroups of RA patients.

\section{Introduction}

Rheumatoid arthritis (RA) is a chronic systemic inflammatory disease characterized by inflammation of the synovia and subsequent destruction of articular structures. It is one of the more common autoimmune disorders causing a significant reduction in healthrelated quality of life $[1,2]$. RA is also associated with a substantial impact on morbidity and mortality leading to high treatment costs and economic burden [3, 4]. Treatment of RA relies primarily on the use of disease-modifying anti-rheumatic drugs (DMARDs). In the last two decades, understanding of the inflammatory process and molecular pathways in RA has increased tremendously. After the breakthrough with tumor necrosis factor inhibitors (TNFi), new biological DMARDs (bDMARDs) with different modes of action targeting several cytokines, B cells, or co-stimulation of lymphocytes were introduced to routine practice showing overall excellent efficacy and acceptable safety in the previous years. However, an important number of patients still fail to reach the target of remission or low disease activity [5]. Therefore, new agents targeting interleukin-6 (IL-6), IL-6 receptor, B cells, IL-17, IL-12/23, IL-20, IL-21, and granulocyte-macrophage colony-stimulating factor (GM-CSF) are in development with a potential to fill the current treatment gap. In this review, we focus on GM-CSF antagonists and summarize the recent clinical trials.

\section{GM-CSF}

GM-CSF is a soluble cytokine responsible for proliferation and differentiation of myeloid cells from bone marrow progenitors. It is a wellknown hemopoietic growth factor used by clinicians for the treatment of neutropenia following chemotherapy. GM-CSF is secreted by numerous cell types including chondrocytes, fibroblasts, activated T cells, and macrophages [6-8]. Pro-inflammatory cytokines including tumor necrosis factor (TNF), IL-1, IL-23, and lipopolysaccharide can stimulate GM-CSF production. Besides neutrophils, activation and survival of eosinophils and macrophages are also regulated by GM-CSF [8, 9]. However, it is also essential in regulating functions of mature myeloid cells such as chemotaxis and cell adhesion, dendritic cell function, expression of pro-inflammatory cytokines, phagocytosis, and microbial killing [10, 11]. GM-CSF can polarize macrophages into M1-like macrophages producing a variety of inflammatory cytokines such as TNF, IL-6, IL-12p70, and IL-23. Therefore, blockade of GM-CSF could favor the polarization into M2-like macrophages, which in contrast produce anti-inflammatory factors such as IL-10 and the chemokine (C-C motif) ligand 2 (CCL2) $[12,13]$. Of note, GM-CSF-deficient mice show no major perturbation of hematopoiesis including number of neutrophils, 
eosinophils, and macrophages, but a defective alveolar macrophage maturation leading to a characteristic pulmonary pathology called pulmonary alveolar proteinosis (PAP) [14]. This is because GM-CSF signaling is crucial in clearing surfactant lipids and proteins from the lung surface by alveolar macrophages. In addition, it promotes the differentiation and pathogenicity of pro-inflammatory T helper 17 cells via IL- 6 and IL-23 $[15,16]$. Furthermore, GM-CSF does not only seem to play a role in causing inflammatory responses, e.g., in joints, but also seem to be involved in pain perception by a separate pathway $[17,18 \bullet \bullet]$.

Figure 1 provides a schematic representation summarizing the association of GM-CSF with cells and cytokines crucial in RA pathogenesis.

GM-CSF receptors are heterodimers composed of a specific-ligand binding $\alpha$-chain (GM-CSFR- $\alpha$ ) and a signal-transducing $\beta$-chain, which is shared with IL-3 and IL-5 receptors $[10,19]$. The cytoplasmic domains of both $\alpha$ and $\beta$ subunits are essential for signal transduction, while the $\beta$ subunit binds to Janus kinase 2 (JAK2) and activates Ras/mitogen-activated protein

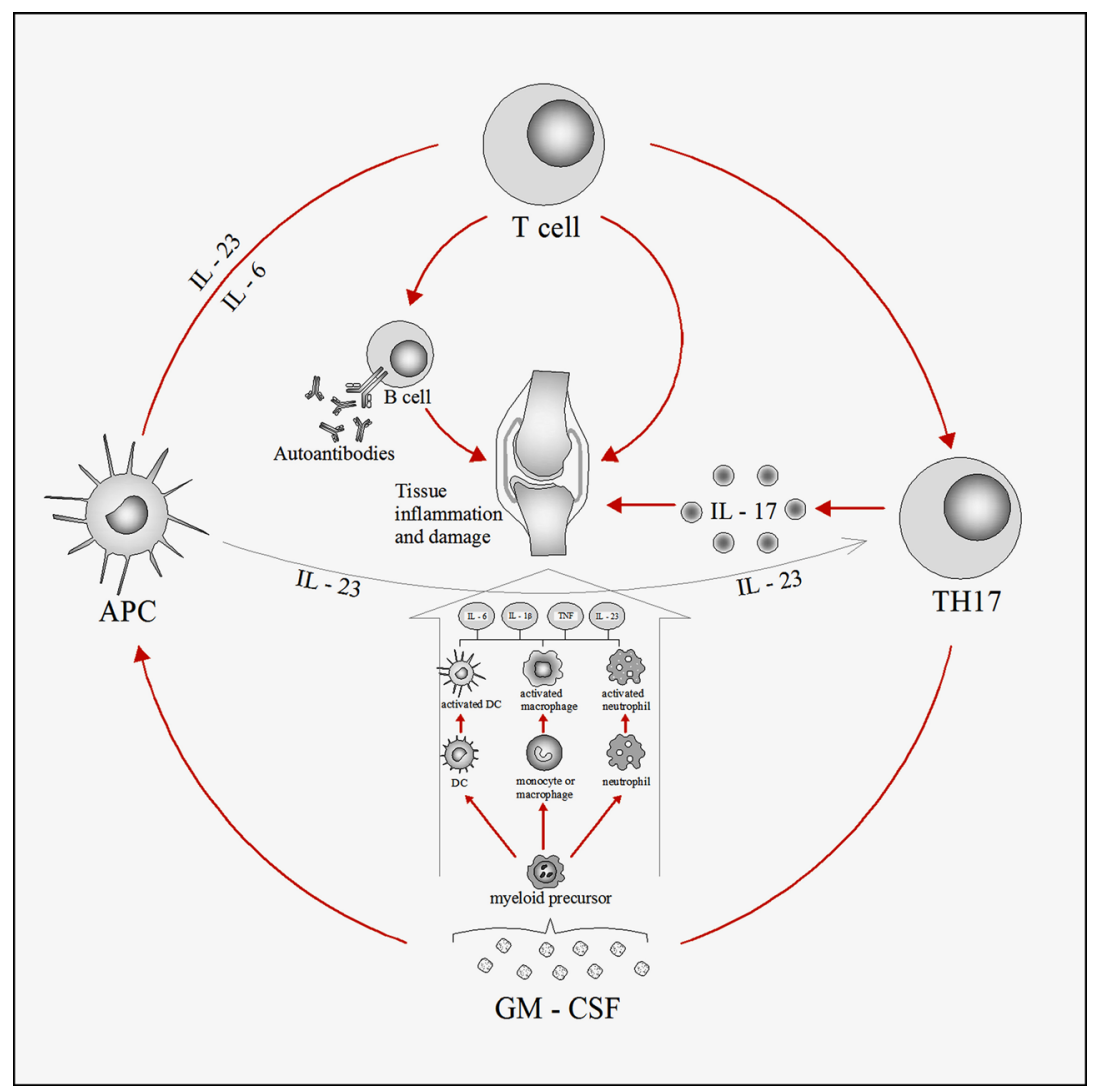

Fig. 1. GM-CSF as a therapeutic target for inflammation. GM-CSF secreted by TH17 cells induces antigen-presenting cells to produce IL-6 and IL-23. IL-6 and IL-23 causes again activation of T cells and differentiation to TH17 cells in a feedback mechanism maintaining the circle. GM-CSF produced by TH17 cells also induces inflammation by activating monocyte-macrophage system and neutrophils. APC antigen-presenting cell, DC dendritic cell, IL interleukin, GM-CSF granulocyte-macrophage colony-stimulating factor, TH17 T helper 17 cell. 
GM-CSF and RA

kinase (MAPK) as well as JAK/signal transducer and activator of transcription (STAT)/suppressor of cytokine signaling (SOCS) signaling [19-21].

Synovial macrophages play an important role in RA-producing crucial cytokines representing the main targets of current therapies such as TNF, IL-1, and IL-6 [22]. GM-CSF and its receptor levels are raised in synovial fluid, plasma, and synoviocytes of RA patients and GM-CSF receptors are overexpressed on circulating mononuclear cells in the synovial tissue [23-26]. Radiographic progression is correlated with number of macrophages in synovial tissue, and reduction of this number is a sensitive biomarker for response to treatment $[27,28]$. These findings suggest a potential role for targeting GM-CSF and its receptors in RA.

\section{Preclinical studies}

Animal models supported an important role for GM-CSF in inflammatory arthritis. While overexpression or direct injection of recombinant GM-CSF causes exacerbation of arthritis, GM-CSF deficiency or blockade of GM-CSF pathway is protective [29-33]. In acute streptococcal cell wall arthritis model (SCWM) of mice, inhibition of GM-CSF suppressed inflammation and protected cartilage [30]. In the same model, TNFi could not alleviate inflammation and inhibit cartilage degradation. Furthermore, IL-1 $\beta$ levels decreased after treatment with GM-CSF monoclonal antibody (mAb) but not in mice receiving TNFi. It was demonstrated that severe inflammatory arthritis can occur in the absence of TNF [34]. Moreover, in clinical practice, an important number of patients do not respond adequately to TNFi [35]. These observations suggest that TNF is not always the primary mediator of RA, and GM-CSF may play a prominent role in TNF-independent disease situations. GMCSF inhibition could also be relevant for combined cytokine blockade, a current issue in treatment of RA. IL-17-/- mice have a reduced level of inflammation compared to wild-type mice in SCWM, and addition of GM-CSF mAb provided further protection in a TNF-independent mouse model [36]. Inhibition of p38 has also been shown to represent an alternative way of reducing TNF release. Some studies provided evidence for a role of GM-CSF in reducing the effectiveness of p38 inhibitor on inhibiting TNF release $[37,38]$. These studies may also form a basis for combined study regimens of GM-CSF and p38 inhibition. In a recently published experimental arthritis study, combined overexpression of IL17A and GM-CSF demonstrated a complementary action and revealed additive and synergistic effects on the production of matrix metalloproteinases (MMPs), receptor activator of nuclear factor (NF)-кB ligand (RANKL), and IL-23 in the synovium which caused a severe destruction of the joint structure in a few days [39••]. In the same study, combined blockade of IL-17 and GM-CSF substantially reduced the joint damage compared to treatment with anti-IL-17 or anti-GM-CSF alone. 


\section{Clinical studies}

Also in humans, flares of arthritis have been observed after administration of recombinant GM-CSF to resolve neutropenia in patients with Felty's syndrome or following chemotherapy supporting the idea of GM-CSF inhibition for treatment of rheumatoid arthritis [40-42].

In light of the preclinical data, clinical studies with molecules targeting GM-CSF itself or its receptor were initiated. Characteristics and current status of these studies are summarized in Table 1.

\section{GM-CSFR inhibition}

\section{First-in-human trial (phase I)}

First-in-human study targeting the innate arm of the immune system via the GM-CSF pathway was performed in patients with mild to moderate RA [43••]. The objective of the trial was to evaluate the safety, tolerability, pharmacokinetic, and pharmacodynamic profiles of mavrilimumab (formerly CAM-3001), a human monoclonal antibody targeting GM-CSFR- $\alpha$, in RA. This was a randomized, double-blind, placebo-controlled, dose-escalating phase I study in subjects with RA who received stable methotrexate (MTX) treatment for $\geq 3$ months before enrolment. The study included men and women aged 18-70 years with a Disease Activity Score 28-joint assessment (DAS28) $\leq 4.8$ (mild to moderate) for $\geq 3$ months. Subjects received a single, escalating intravenous dose of mavrilimumab (0.01,0.03, 0.1, 0.3, 1.0, 3.0, and $10.0 \mathrm{mg} /$ $\mathrm{kg}$ ) or placebo on study day 0 and were followed up for 24 weeks. Patients continued their stable dose of MTX. In total, 32 subjects were enrolled and completed planned evaluations including safety analyses. The peak plasma concentrations (Cmax) were 0.241 and $373 \mu \mathrm{g} / \mathrm{ml}$ at doses of 0.01 and $10 \mathrm{mg} / \mathrm{kg}$, respectively. Systemic clearance decreased from 88.8 to $3.15 \mathrm{ml} / \mathrm{kg} /$ day, when the dose was increased from 0.01 to $10.0 \mathrm{mg} / \mathrm{kg}$. The elimination half-lives at 0.1 and $10 \mathrm{mg} / \mathrm{kg}$ were 1.81 and 15.1 days, respectively. Four hours after dosing, the induction of SOCS3 mRNA by GM-CSF was significantly inhibited with doses of $1.0 \mathrm{mg} / \mathrm{kg}$ and this effect was sustained during 2 weeks.

Adverse events (AEs) were mostly mild, unrelated to drug dosage and similar between the mavrilimumab and placebo cohorts. The most commonly reported AEs were nasopharyngitis, headache, diarrhea, and back pain. Only one significant episode of face and neck urticaria was reported in a subject at $10.0 \mathrm{mg} / \mathrm{kg}$ cohort, which led to early termination of the infusion. No significant hematological changes or abnormalities in lung function were noted.

The study was not powered to demonstrate efficacy and $63 \%$ of patients had normal acute phase reactants at baseline. Although no significant change in DAS2 8 were observed in any of the cohorts, patients with moderate disease activity at baseline showed a significant reduction of DAS28 at 4 weeks. Moreover, reductions of acute phase reactants was observed in subjects with elevated erythrocyte 


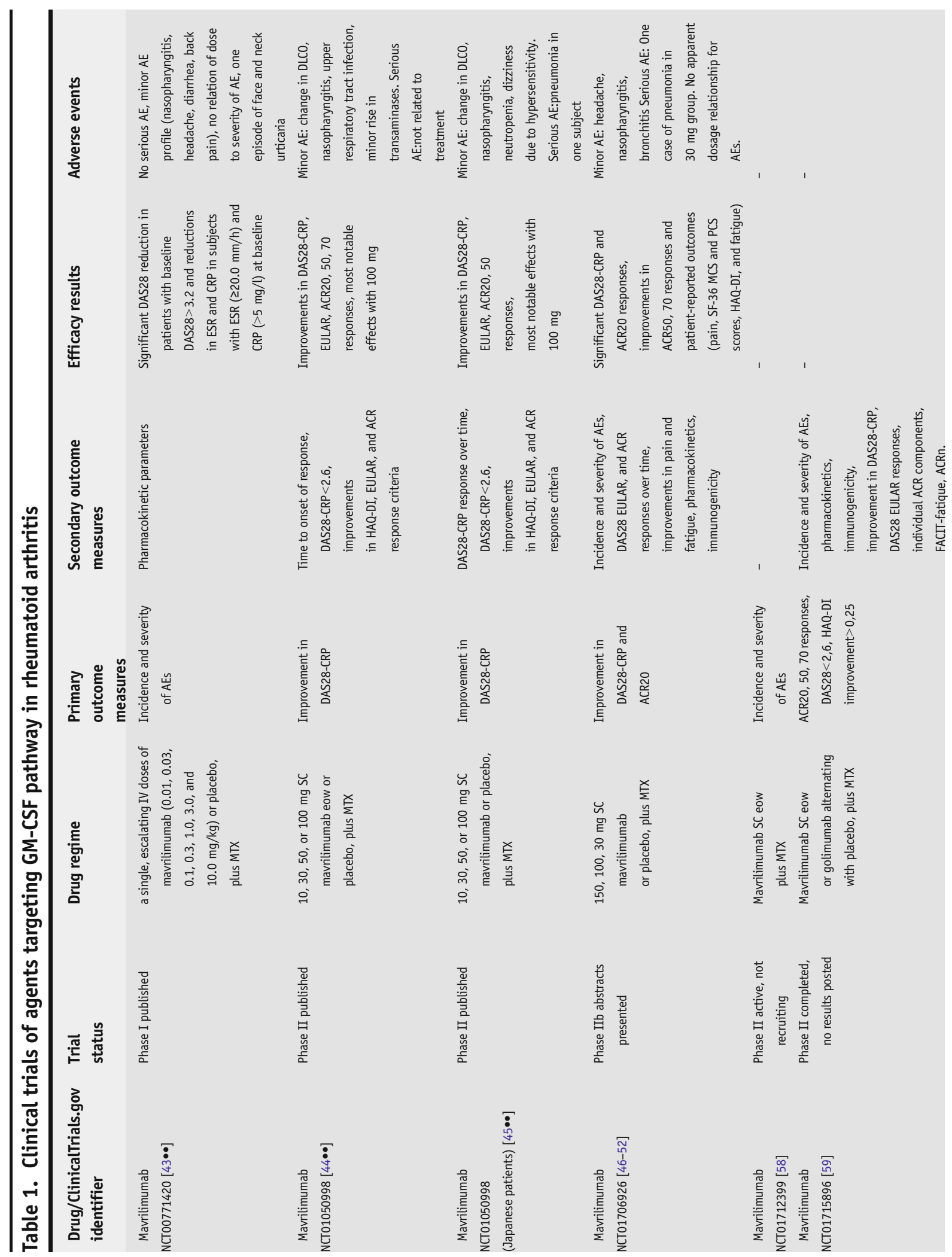




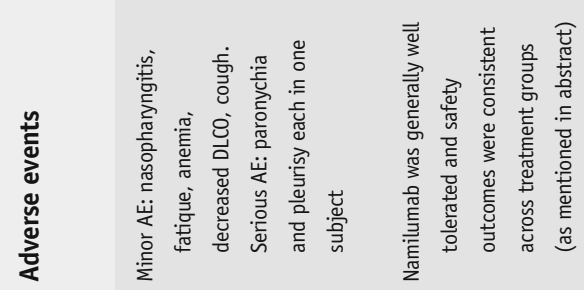

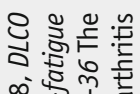

柁

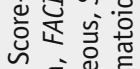

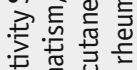

害咢言

嵌

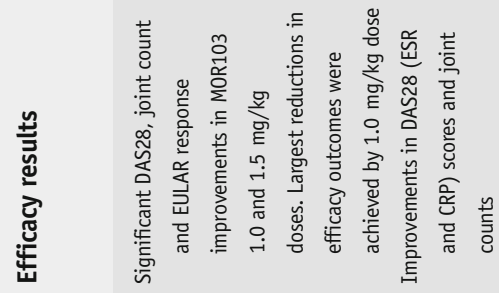

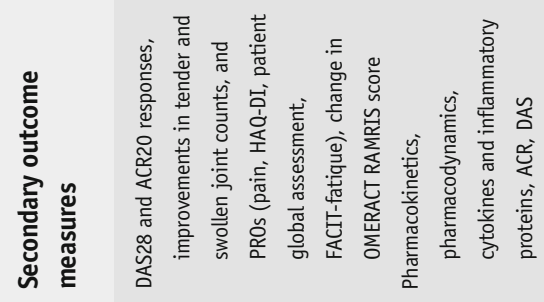

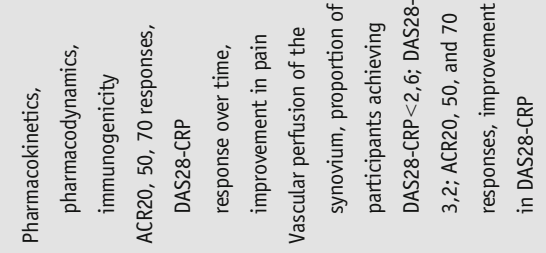

芒范

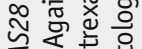

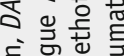

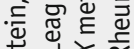

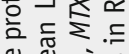

$\overbrace{0}^{\circ}$

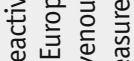

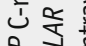

灾宓济

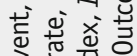

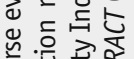

辛空:

岸.

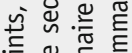

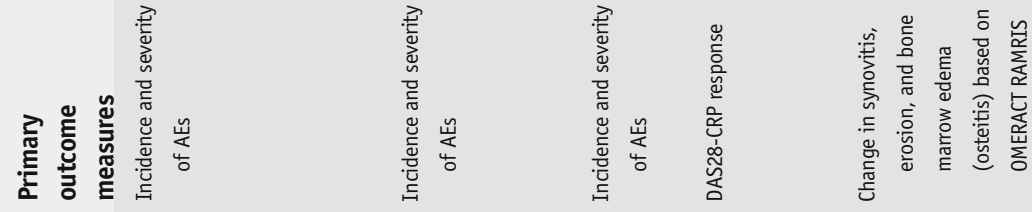

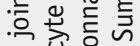

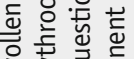

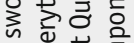

든

㐫进氙.

这苍匍

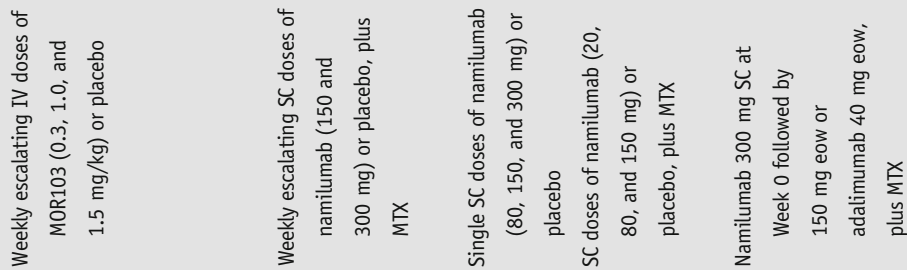

㐫辛苋

至视京

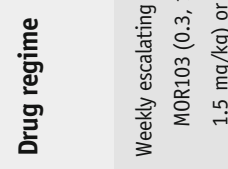
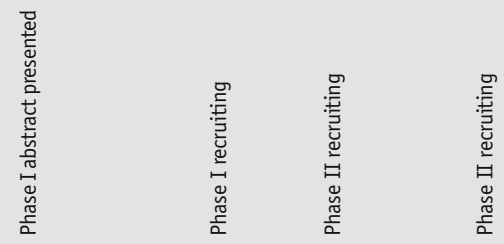

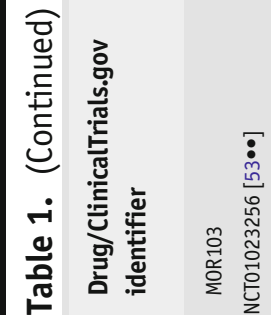
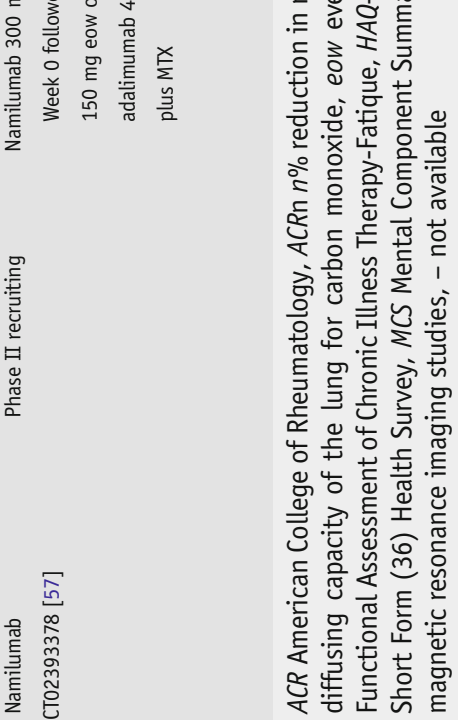
sedimentation rate (ESR) $(\geq 20.0 \mathrm{~mm} / \mathrm{h})$ and C-reactive protein (CRP) $(>5 \mathrm{mg} / \mathrm{l})$ at baseline. In summary, the obtained safety and pharmacokinetic profiles encouraged further trials with mavrilimumab.

\section{Phase II trials}

The phase II randomized, double-blind placebo-controlled study (EARTH) of mavrilimumab aimed to investigate the efficacy and safety in 233 subjects with

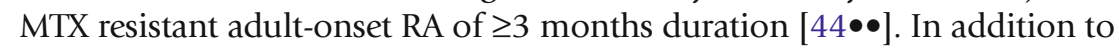
stable MTX dosages, subcutaneous mavrilimumab $(10,30,50$, or $100 \mathrm{mg})$ or placebo was administered every other week (eow) for 12 weeks and patients were followed for additional 12 weeks. The proportion of patients achieving the primary endpoint, a decrease in DAS28-CRP $\geq 1.2$ at week 12 , was significantly higher in mavrilimumab-treated group compared to placebo (55.7 vs $34.7 \%$, $p=0.003$ ). Observation of differences in response rates started at week 2 and increased throughout the treatment period. Most significant effects were seen with the highest dose of $100 \mathrm{mg}$ with a DAS28-CRP $<2.6$ (23.1 vs $6.7 \%$, $p=$ 0.016). Furthermore, all categories of the American College of Rheumatology (ACR) criteria (ACR20 69.2 vs $40.0 \%, p=0.005$; ACR50 30.8 vs $12.0 \%, p=$ 0.021 ; ACR70 17.9 vs $4.0 \%, p=0.030$ ), as well as the Health Assessment Questionnaire Disability Index (HAQ-DI) $(-0.48$ vs $-0.25, p=0.005)$ were improved in 100-mg group. Significantly higher number of subjects on mavrilimumab showed a moderate or good European League Against Rheumatism (EULAR) response versus placebo ( 67.7 vs $50.7 \%, p=0.025)$, and the highest proportion of moderate (46.2\%) or good EULAR responders (30.8\%) was seen again in the 100-mg group. Significant changes in ESR and CRP were evident by week 2 and in tender and swollen joint counts by week 4 . Efficacy observed at week 12 was maintained during the 12 weeks of follow-up period for $100 \mathrm{mg}$ mavrilimumab in terms of primary endpoint and ACR20. This "post-treatment" maintenance of response was not observed for DAS28-CRP remission (<2.6), ACR50, and ACR70 responses. Adverse events were generally mild or moderate consistent with the phase I study. There was not any significant hypersensitivity reaction, serious or opportunistic infection. Importantly, there were also no clinically significant or persistent changes in lung function tests. Biomarker assessments reflecting inflammation and cartilage degradation were performed showing decreased levels of acute phase proteins and IL- 6 within 1 month. This suggests a direct effect on IL-6, while observed changes in MMP3 and YKL-40 could indicate a beneficial effect on cartilage degradation. The confirmed rapid clinically significant responses supported new trials with higher number of patients.

As add-on to the EARTH study, another study was performed in adult Japanese subjects with moderate to severe RA [45••]. Of note, it was not powered for a separate analysis of the Japanese population. Fifty-one subjects received 10,30, 50, or $100 \mathrm{mg}$ of mavrilimumab SC or placebo every other week for 12 weeks, followed by a 12-week follow-up period. Patients also continued weekly MTX intake. As a result, a higher proportion of the mavrilimumab-treated patients achieved the primary endpoint of $>1.2$ reduction in DAS28-CRP compared to placebo at week 12 (50.0 vs $23.5 \%, p=0.081$ ). The 30- and 100-mg dose groups reached significant responses with respect to 
the primary outcome (both 75.0 vs $23.5 \%, p=0.028$ ). In this context, it should be considered that mean DAS28-CRP and tender and swollen joint counts were lower in the 50-mg group. The 100-mg dose group demonstrated significant difference already by week 6 , while 30-mg dose group demonstrated effects by week 8 . Although remission rates (DAS28-CRP $<2.6$ points) were numerically higher in mavrilimumab-treated subjects, this did not reach statistical significance. The 100-mg dose group had significantly higher ACR 20 responses by week 4 and a significantly greater HAQ-DI mean difference by week 2 compared to placebo. The 100-mg dose group also demonstrated a considerable ACR50 response versus placebo at week 12 ( 50.0 vs $11.8 \%, p=0.059$ ), but none of these patients achieved ACR70 response. In comparison with European and general data, 100-mg dose group showed high degree of consistency in almost all efficacy end points, with the exception of ACR70. Safety, tolerability, and pharmacokinetics data were also consistent with European data and AEs were mild or moderate. There was only one serious $\mathrm{AE}$ of pneumonia possibly related to the study medication. Taken together, although the study was not powered to evaluate efficacy in the Japanese cohort, positive trends were noted for various endpoints. As in the European cohort, best results were obtained with $100 \mathrm{mg}$ dose and the proportion of subjects responding to mavrilimubab increased throughout the 12-week with maintenance of response also during the follow-up period.

Results of phase IIb study (EARTH EXPLORER 1) were presented recently as abstracts [46-52]. The aim of the study was to evaluate the efficacy and safety of mavrilimumab in moderate to severe (DAS28-CRP $\geq 3.2 ; \geq 4$ swollen joint) RA patients with an inadequate response to $\geq 1$ DMARDs. In this 24 -week study, patients aged 18-80 years were randomized to receive one of three SC mavrilimumab dosages (150, 100, $30 \mathrm{mg}$ eow) or placebo plus MTX (7.5$25.0 \mathrm{mg} /$ week). Primary endpoints were change in DAS28-CRP at week 12 and ACR20 response at week 24 . At week 12, all doses of mavrilimumab demonstrated a significant $(p<0.001)$ DAS28-CRP response versus placebo. Also at week 24, a statistically significant ACR20 response $(p<0.001)$ was observed for all dosages of mavrilimumab vs placebo. Moreover, all mavrilimumab groups achieved significant ACR50 response compared to placebo; however, only the 150 -mg dose demonstrated a significant ACR70 response vs placebo. The most common treatment-emergent AEs were headache (7.6, 4.7, 6.2, $2.5 \%)$,

nasopharyngitis $(7.6,3.5,4.9,7.4 \%)$, and bronchitis $(5.1,1.2,3.7,7.4 \%)$ for mavrilimumab 150, 100, and $30 \mathrm{mg}$ eow or placebo, respectively. Pulmonary AEs were not higher compared to placebo $(6.3,3.5$, and $6.2 \%$ vs $9.9 \%)$. Two cases of pneumonia were observed, one in the placebo group and one in the 30$\mathrm{mg}$ group. There was no apparent dosage relationship for AEs. Greater than $90 \%$ of patients entered a long-term, open-label extension study. In summary, mavrilimumab was generally well tolerated and met the primary endpoints with a clear dose response. Treatment effect was evident across several secondary endpoints compared to placebo already after the first week. Considering patient-reported outcomes (PROs), all mavrilimumab treatment groups exhibited improvements in pain, SF-36 Mental Component Summary (MCS) and Physical Component Summary (PCS) scores, physical function (HAQ-DI) and fatigue (FACIT-F) vs. placebo at weeks 12 and 24. At the $150 \mathrm{mg}$ dosage, significant improvements were demonstrated across all PRO endpoints ( $p=$ 0.019 to $p<0.001$ vs. placebo) at weeks 12 and 24, except for SF-36 MCS (week 


\section{GM-CSF inhibition}

$12, p=0.007$; week $24, p=0.187)$. Statistically significant improvements in pain were demonstrated very early in mavrilimumab groups beginning at week 1 . Improvements in pain and physical function were sustained in the majority of mavrilimumab-treated patients at week 12 through week 24. Analysis of peripheral markers and pathophysiologic pathways indicated that the sustained efficacy may result also from indirect effects on $\mathrm{T}$ cell activation in addition to direct effects on macrophages. The demonstrated suppression of citrullination and extracellular matrix degradation suggests an efficacy of mavrilimumab therapy on tissue damage.

\section{MOR103}

In addition to mavrilimumab, a human monoclonal antibody to GM-

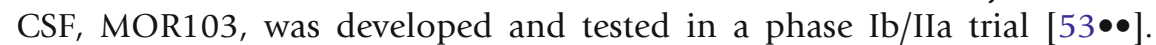
The aim of the study was to determine the safety, tolerability, and signs of efficacy in a randomized, double-blind, placebo-controlled dose-escalation design. Intravenous MOR103 was administrated at three doses $(0.3,1.0$, and $1.5 \mathrm{mg} / \mathrm{kg})$ once a week for 4 weeks with a follow-up period of 16 weeks in patients with active moderate RA. Ninety-six subjects were randomized and treated and 85 of them completed the trial $(n=27,24,22$, and 23 for pooled placebo and MOR103 0.3, 1.0, and $1.5 \mathrm{mg} / \mathrm{kg}$, respectively). As in previous studies with mavrilimumab, AEs were generally mild or moderate in intensity, while the most common AE was nasopharyngitis. There were two treatment-related events rated as serious because of hospitalization, a paronychia in the placebo group and a pleurisy in the MOR103-treated group, both recovered fully. A decrease in DLCO in a patient under placebo led to treatment discontinuation. In exploratory investigations, MOR103 1.0 and $1.5 \mathrm{mg} / \mathrm{kg}$ treatments were associated with significant improvements in DAS28 scores, joint counts, and EULAR response rates compared to placebo. Improvements in DAS28 scores could be observed from the first week on. Largest reductions in DAS28 scores and additional efficacy outcomes were achieved by MOR103 $1.0-\mathrm{mg} / \mathrm{kg}$ group. Only the response to the 1 $\mathrm{mg} / \mathrm{kg}$ dose was maintained until week 16 . These data also encourages further investigations with this monoclonal antibody to GM-CSF in RA.

Namilumab (MT203), another anti-GM-CSF monoclonal antibody, has been evaluated in a phase Ib study, PRIORA [54]. In this double-blind, placebocontrolled, randomized, dose-escalating study, patients with mild to moderate RA on stable MTX doses were included. The treatment regimen consisted of three single injections of namilumab 150 or $300 \mathrm{mg}$ or matching placebo on days 0,15 , and 29, with 12 weeks of follow-up. As in previous studies targeting GM-CSF pathway, namilumab was generally well tolerated and safety outcomes were similar across the treatment groups. A greater improvements in DAS28 (ESR and CRP) scores and joint counts could be observed in patients treated with namilumab compared to placebo at day 29. In addition, more namilumab patients $(10 / 14,71.4 \%)$ had a DAS28-ESR response ( $>1.2$ decrease from 


\section{Conclusion}

baseline) than placebo patients $(2 / 7,28.6 \%)$ at day 56 (4 weeks after the last study dose). Another phase I and two phase II trials of namilumab are already recruiting [55-57].

Published studies suggest that GM-CSF inhibition offers a new and effective strategy in treatment of RA. The phase II studies confirmed early and sustained effects and a favorable safety. However, efficacy needs to be evaluated in larger numbers of patients both in conventional DMARD and anti-TNF resistant patients. A long-term safety trial is still ongoing and a head-to-head study with a TNF-inhibitor has been completed but not published so far [58, 59]. Consistency of results both in European and Japanese cohorts also supports worldwide phase III trials. In addition to GM-CSFR blockade, also inhibition of GMCSF itself seems to have similar effects as shown by first studies. As shown by preclinical studies, GM-CSF inhibition offers a promising approach in TNFindependent manifestations and could be used in combination with other targeted therapies. Finally, it will be a major task to find the optimal position for this treatment approach in the current algorithms for RA treatment.

\section{Compliance with Ethics Guidelines}

\section{Conflict of Interest}

Dr. Avci declares that he has no conflict of interest. Dr. Feist declares that he has no conflict of interest. Dr. Burmester reports personal fees from Medlmmune during the conduct of the study.

\section{Human and Animal Rights and Informed Consent}

This article does not contain any studies with human or animal subjects performed by any of the authors.

\section{References}

Papers of particular interest have been

highlighted as:

- Of major importance

1. Cross M, Smith E, Hoy D, Carmona L, Wolfe F, Vos T, et al. The global burden of rheumatoid arthritis: estimates from the global burden of disease 2010 study. Ann Rheum Dis. 2014;73(7):1316-22.

2. Matcham F, Scott IC, Rayner L, Hotopf M, Kingsley GH, Norton S, et al. The impact of rheumatoid arthritis on quality-of-life assessed using the SF-36: a systematic review and meta-analysis. Semin Arthritis Rheum. 2014;44(2):123-30.

3. Hulsemann JL, Mittendorf T, Merkesdal S, Handelmann S, von der Schulenburg JM, Zeidler H, et al. Direct costs related to rheumatoid arthritis: the patient perspective. Ann Rheum Dis. 2005;64(10):1456-61.

4. Sokka T, Kautiainen H, Pincus T, Verstappen SM, Aggarwal A, Alten R, et al. Work disability remains a major problem in rheumatoid arthritis in the 2000s: data from 32 countries in the QUEST-RA study. Arthritis Res Ther. 2010;12(2):R42.

5. Smolen JS, Aletaha D. Rheumatoid arthritis therapy reappraisal: strategies, opportunities and challenges. Nat Rev Rheumatol. 2015;11(5):276-89. 
6. Leizer T, Cebon J, Layton JE, Hamilton JA. Cytokine regulation of colony-stimulating factor production in cultured human synovial fibroblasts: I. Induction of GM-CSF and G-CSF production by interleukin-1 and tumor necrosis factor. Blood. 1990;76(10):1989-96.

7. Campbell IK, Novak U, Cebon J, Layton JE, Hamilton JA. Human articular cartilage and chondrocytes produce hemopoietic colony-stimulating factors in culture in response to IL-1. J Immunol. 1991;147(4):1238-46.

8. Hamilton JA. Colony-stimulating factors in inflammation and autoimmunity. Nat Rev Immunol.

2008;8(7):533-44.

9. Cheung DL, Hamilton JA. Regulation of human monocyte DNA synthesis by colony-stimulating factors, cytokines, and cyclic adenosine monophosphate. Blood. 1992;79(8):1972-81.

10. Fleetwood AJ, Cook AD, Hamilton JA. Functions of granulocyte-macrophage colony-stimulating factor. Crit Rev Immunol. 2005;25(5):405-28.

11. Cook AD, Braine EL, Hamilton JA. Stimulus-dependent requirement for granulocyte-macrophage colony-stimulating factor in inflammation. J Immunol.

2004;173(7):4643-51.

12. Fleetwood AJ, Lawrence T, Hamilton JA, Cook AD. Granulocyte-macrophage colony-stimulating factor (CSF) and macrophage CSF-dependent macrophage phenotypes display differences in cytokine profiles and transcription factor activities: implications for CSF blockade in inflammation. J Immunol.

2007;178(8):5245-52.

13. Mantovani A, Sozzani S, Locati M, Allavena P, Sica A. Macrophage polarization: tumor-associated macrophages as a paradigm for polarized M2 mononuclear phagocytes. Trends Immunol. 2002;23(11):549-55.

14. Stanley E, Lieschke GJ, Grail D, Metcalf D, Hodgson G, Gall JA, et al. Granulocyte/macrophage colonystimulating factor-deficient mice show no major perturbation of hematopoiesis but develop a characteristic pulmonary pathology. Proc Natl Acad Sci U S A. 1994;91(12):5592-6.

15. Codarri L, Gyulveszi G, Tosevski V, Hesske L, Fontana A, Magnenat L, et al. RORgammat drives production of the cytokine GM-CSF in helper T cells, which is essential for the effector phase of autoimmune neuroinflammation. Nat Immunol. 2011;12(6):560-7.

16. El-Behi M, Ciric B, Dai H, Yan Y, Cullimore M, Safavi F, et al. The encephalitogenicity of $\mathrm{T}(\mathrm{H}) 17$ cells is dependent on IL-1- and IL-23-induced production of the cytokine GM-CSF. Nat Immunol. 2011;12(6):568-75.

17. Cook AD, Pobjoy J, Steidl S, Durr M, Braine EL, Turner $\mathrm{AL}$, et al. Granulocyte-macrophage colony-stimulating factor is a key mediator in experimental osteoarthritis pain and disease development. Arthritis Res Ther. 2012;14(5):R199.

18.• Cook AD, Pobjoy J, Sarros S, Steidl S, Durr M, Lacey DC, et al. Granulocyte-macrophage colony-stimulating factor is a key mediator in inflammatory and arthritic pain. Ann Rheum Dis. 2013;72(2):265-70.
In this experimental study, besides causing inflammatory responses GM-CSF also seemed to be involved in pain development. Results of the study suggest that, in addition to inflammation control GM-CSF inhibition may alleviate pain by a separate pathway in inflammatory arthritis.

19. Hansen G, Hercus TR, McClure BJ, Stomski FC, Dottore $\mathrm{M}$, Powell J, et al. The structure of the GM-CSF receptor complex reveals a distinct mode of cytokine receptor activation. Cell. 2008;134(3):496-507.

20. Jenkins BJ, Blake TJ, Gonda TJ. Saturation mutagenesis of the beta subunit of the human granulocytemacrophage colony-stimulating factor receptor shows clustering of constitutive mutations, activation of ERK MAP kinase and STAT pathways, and differential beta subunit tyrosine phosphorylation. Blood. 1998;92(6):1989-2002.

21. Sato N, Sakamaki K, Terada N, Arai K, Miyajima A. Signal transduction by the high-affinity GM-CSF receptor: two distinct cytoplasmic regions of the common beta subunit responsible for different signaling. EMBO J. 1993;12(11):4181-9.

22. Brennan FM, McInnes IB. Evidence that cytokines play a role in rheumatoid arthritis. J Clin Invest.

2008;118(11):3537-45.

23. Bell AL, Magill MK, McKane WR, Kirk F, Irvine AE. Measurement of colony-stimulating factors in synovial fluid: potential clinical value. Rheumatol Int. 1995; 14(5):177-82.

24. Fiehn C, Wermann M, Pezzutto A, Hufner M, Heilig B. Plasma GM-CSF concentrations in rheumatoid arthritis, systemic lupus erythematosus and spondyloarthropathy. Z Rheumatol. 1992;51(3):1216. GM-CSF-Plasmakonzentrationen bei rheumatoider Arthritis, systemischem Lupus erythematodes und Spondylarthropathie.

25. Berenbaum F, Rajzbaum G, Amor B, Toubert A. Evidence for GM-CSF receptor expression in synovial tissue. An analysis by semi-quantitative polymerase chain reaction on rheumatoid arthritis and osteoarthritis synovial biopsies. Eur Cytokine Netw. 1994;5(1):43-6.

26. Davis 3rd JM, Knutson KL, Strausbauch MA, Crowson CS, Therneau TM, Wettstein PJ, et al. Analysis of complex biomarkers for human immune-mediated disorders based on cytokine responsiveness of peripheral blood cells. J Immunol. 2010;184(12):7297-304.

27. Mulherin D, Fitzgerald O, Bresnihan B. Synovial tissue macrophage populations and articular damage in rheumatoid arthritis. Arthritis Rheum. 1996;39(1):115-24.

28. Haringman JJ, Gerlag DM, Zwinderman AH, Smeets TJ, Kraan MC, Baeten D, et al. Synovial tissue macrophages: a sensitive biomarker for response to treatment in patients with rheumatoid arthritis. Ann Rheum Dis. 2005;64(6):834-8.

29. Cook AD, Braine EL, Campbell IK, Rich MJ, Hamilton JA. Blockade of collagen-induced arthritis post-onset by antibody to granulocyte-macrophage colony- 
stimulating factor (GM-CSF): requirement for GM-CSF in the effector phase of disease. Arthritis Res. 2001;3(5):293-8.

30. Plater-Zyberk C, Joosten LA, Helsen MM, Hepp J, Baeuerle PA, van den Berg WB. GM-CSF neutralisation suppresses inflammation and protects cartilage in acute streptococcal cell wall arthritis of mice. Ann Rheum Dis. 2007;66(4):452-7.

31. Campbell IK, Bendele A, Smith DA, Hamilton JA. Granulocyte-macrophage colony stimulating factor exacerbates collagen induced arthritis in mice. Ann Rheum Dis. 1997;56(6):364-8.

32. Lang RA, Metcalf D, Cuthbertson RA, Lyons I, Stanley E, Kelso A, et al. Transgenic mice expressing a hemopoietic growth factor gene (GM-CSF) develop accumulations of macrophages, blindness, and a fatal syndrome of tissue damage. Cell. 1987;51(4):675-86.

33. Campbell IK, Rich MJ, Bischof RJ, Dunn AR, Grail D, Hamilton JA. Protection from collagen-induced arthritis in granulocyte-macrophage colony-stimulating factordeficient mice. J Immunol. 1998;161(7):3639-44.

34. Campbell IK, O'Donnell K, Lawlor KE, Wicks IP. Severe inflammatory arthritis and lymphadenopathy in the absence of TNF. J Clin Invest. 2001;107(12):1519-27.

35. Solomon DH, Bitton A, Katz JN, Radner H, Brown EM, Fraenkel L. Review: treat to target in rheumatoid arthritis: fact, fiction, or hypothesis? Arthritis Rheumatol. 2014;66(4):775-82.

36. Plater-Zyberk C, Joosten LA, Helsen MM, Koenders MI, Baeuerle PA, van den Berg WB. Combined blockade of granulocyte-macrophage colony stimulating factor and interleukin 17 pathways potently suppresses chronic destructive arthritis in a tumour necrosis factor alphaindependent mouse model. Ann Rheum Dis. 2009;68(5):721-8.

37. Hua F, Henstock PV, Tang B. ERK activation by GMCSF reduces effectiveness of p38 inhibitor on inhibiting TNFalpha release. Int Immunopharmacol. 2010;10(7):730-7.

38. Espelin CW, Goldsipe A, Sorger PK, Lauffenburger DA, de Graaf D, Hendriks BS. Elevated GM-CSF and IL1beta levels compromise the ability of p38 MAPK inhibitors to modulate TNFalpha levels in the human monocytic/macrophage U937 cell line. Mol BioSyst. 2010;6(10):1956-72.

39.•• van Nieuwenhuijze AE, van de Loo FA, Walgreen B, Bennink M, Helsen M, van den Bersselaar L, et al. Complementary action of granulocyte-macrophage colony-stimulating factor and interleukin-17A induces interleukin-23, receptor activator of nuclear factor-kappaB ligand and matrix metalloproteinases and drives bone and cartilage pathology in experimental arthritis: rationale for combination therapy in rheumatoid arthritis. Arthritis Res Therapy. 2015;17(1):163.

In this recently published experimental arthritis study, combined overexpression of IL-17A and GM-CSF demonstrated additive and synergistic effects with severe destruction of the joint structure and combined blockade of these two cytokines substantially reduced the joint damage compared to treatment with anti-IL-17 or anti-GM-CSF alone. Therefore, comprehensive assessment of GM-CSF in combination strategies will be of major importance.

40. Hazenberg BP, Van Leeuwen MA, Van Rijswijk MH, Stern AC, Vellenga E. Correction of granulocytopenia in Felty's syndrome by granulocyte-macrophage colony-stimulating factor. Simultaneous induction of interleukin- 6 release and flare-up of the arthritis. Blood. 1989;74(8):2769-70.

41. Pereira J, Velloso ED, Loterio HA, Laurindo IM, Chamone DA. Long-term remission of neutropenia in Felty's syndrome after a short GM-CSF treatment. Acta Haematol. 1994;92(3):154-6.

42. de Vries EG, Willemse PH, Biesma B, Stern AC, Limburg PC, Vellenga E. Flare-up of rheumatoid arthritis during GM-CSF treatment after chemotherapy. Lancet. 1991;338(8765):517-8.

43.•• Burmester GR, Feist E, Sleeman MA, Wang B, White B, Magrini F. Mavrilimumab, a human monoclonal antibody targeting GM-CSF receptor-alpha, in subjects with rheumatoid arthritis: a randomised, double-blind, placebo-controlled, phase I, first-in-human study. Ann Rheum Dis. 2011;70(9):1542-9.

This is the first-in-human study targeting the innate arm of the immune system via the GM-CSF pathway. The obtained favorable safety and pharmacokinetic profiles with GM-CSFR $\mathrm{mAb}$ encouraged further trials with mavrilimumab.

44.• Burmester GR, Weinblatt ME, McInnes IB, Porter D, Barbarash O, Vatutin M, et al. Efficacy and safety of mavrilimumab in subjects with rheumatoid arthritis. Ann Rheum Dis. 2013;72(9):1445-52.

This phase II study of mavrilimumab demonstrated profound and rapid onset of response, normalisation of acute phase reactants (CRP and ESR) with a good safety data. This observation suggests that inhibiting the mononuclear phagocyte pathway may provide a novel therapeutic approach for RA.

45.• Takeuchi T, Tanaka Y, Close D, Godwood A, Wu CY, Saurigny D. Efficacy and safety of mavrilimumab in Japanese subjects with rheumatoid arthritis: findings from a phase IIa study. Modern rheumatology/the Japan Rheumatism Association. 2015;25(1):21-30. Epub 2014/04/12.

Results of this study in Japanese subjects also demonstrated rapid and clinically meaningful efficacy with a good safety data consistent with European cohort. This observation supports worldwide phase III trials.

46. Burmester GR, McInnes IB, Kremer JM, Miranda P, Korkosz M, Vencovsky J, et al. Efficacy and safety of mavrilimumab, a fully human GM-CSFR-alpha monoclonal antibody in patients with rheumatoid arthritis: primary results from the EARTH EXPLORER 1 study. EULAR 2015: Ann Rheum Dis. 2015;74(2):78.

47. Kremer JM, Burmester GR, Weinblatt M, Williams AE, Karlsson N, Godwood A, et al. Patient-reported outcomes (PROS) during treatment with mavrilimumab, a fully human monoclonal antibody targeting GMCSFR-alpha, in the phase IIb EARTH EXPLORER 1 
study. EULAR 2015. Ann Rheum Dis.

2015;74(Suppl2):483.

48. McInnes IB, Burmester GR, Kremer JM, Miranda P, Korkosz M, Vencovsky J, et al. Rapid onset of clinical benefit in patients with RA treated with mavrilimumab, a fully human monoclonal antibody targeting GM-CSFR-alpha: subanalysis of the phase IIb EARTH EXPLORER 1 study. EULAR 2015: Ann Rheum Dis. 2015;74(Suppl2):723.

49. Guo X, Sinibaldi D, Kuziora M, Brohawn P, Ryan PC, Bay-Jensen AC, et al. Sustained response to mavrilimumab in rheumatoid arthritis patients via suppression of macrophage and T cells. EULAR 2015. Ann Rheum Dis. 2015;74(Suppl2):734.

50. Jin DC, Wu CY, Roskos LK, Godwood A, Close D, Wang B. Exposure-efficacy analysis of mavrilimumab in rheumatoid arthritis: modeling and simulation of phase II clinical data. EULAR 2015. Ann Rheum Dis. 2015;74(Suppl2):1043.

51. Kremer JM, Burmester GR, Weinblatt M, Williams AE, Karlsson N, Godwood A, et al. Analysis of patientreported outcomes during treatment with mavrilimumab, a human monoclonal antibody targeting GM-CSFRá, in the randomized phase $2 \mathrm{~b}$ earth explorer 1 study. ACR 2014.

52. McInnes IB, Burmester GR, Kremer JM, Miranda P, Korkosz M., Vencovsky J, et al. Rapid onset of clinical benefit is associated with a reduction in validated biomarkers of disease in patients with rheumatoid arthritis treated with mavrilimumab, a human monoclonal antibody targeting GM-CSFRÃ $\tilde{i}_{j}$. ACR 2014.

53.• Behrens F, Tak PP, Ostergaard M, Stoilov R, Wiland P, Huizinga TW, et al. MOR103, a human monoclonal antibody to granulocyte-macrophage colony-stimulating factor, in the treatment of patients with moderate rheumatoid arthritis: results of a phase $\mathrm{Ib} / \mathrm{IIa}$ randomised, double-blind, placebo-controlled, dose-escalation trial. Ann Rheum Dis. 2015;74(6):1058-64.

This is the first-in-human study targeting GM-CSF itself instead of GM-CSFR. Good tolerability and preliminary evidence of efficacy supports further trials with this monoclonal antibody.

54. Huizinga TWJ, Batalov A, Yablanski K, Stoilov R, Lloyd E, Wagner T, et al. First-in-patient study of namilumab, an anti-GM-CSF monoclonal antibody, in active rheumatoid arthritis: results of the PRIORA phase Ib study. EULAR 2015. Ann Rheum Dis. 2015;74(Suppl2):733.

55. A Phase 1 MT203 single-dose study to evaluate safety, PK and PD. ClinicalTrials.gov [August 1, 2015]; Available from: https://clinicaltrials.gov/ct2/show/ NCT02354599?term=namilumab\&rank=1.

56. Dose finding study of namilumab in combination with methotrexate in participants with moderate to severe rheumatoid arthritis (RA). ClinicalTrials.gov [August 1, 2015]; Available from: https://clinicaltrials.gov/ct2/ show/NCT02379091?term=namilumab\&rank=3.

57. Namilumab vs adalimumab in participants with moderate to severe early rheumatoid arthritis inadequately responding to methotrexate (TELLUS). ClinicalTrials.gov [August 1, 2015]; Available from: https://clinicaltrials.gov/ct2/show/NCT02393378? term=namilumab\&rank $=4$.

58. A long term safety study of Mavrilimumab in adult subjects with rheumatoid Arthritis. ClinicalTrials.gov [August 1, 2015]; Available from: https://clinicaltrials. gov/ct2/show/NCT01712399?term= mavrilimumab\&rank $=4$.

59. A study of mavrilimumab versus anti tumor necrosis factor in subjects with rheumatoid arthritis. ClinicalTrials.gov [August 1, 2015]; Available from: https://cinicaltrials.gov/ct2/show/study/ NCT01715896?term=mavrilimumab\&rank=3. 